\title{
Multidisciplinary Novel Approach with the Aid of Ideal Denture Setup throughout Treatment Steps of Complete Implant Rehabilitation
}

\author{
Mohammed E. Sayed ${ }^{1,2 *}$, Christopher J. Rochford ${ }^{3}$, Barry E. Zweig ${ }^{3}$ and Robert J. Flinton ${ }^{4}$ \\ ${ }^{1}$ Department of Prosthetic Dental Sciences, Jazan University Faculty of Dentistry, Jazan, Saudi Arabia \\ ${ }^{2}$ Department of Restorative Dentistry, Rutgers School of Dental Medicine, Newark, New Jersey, USA \\ ${ }^{3}$ Department of Oral and Maxillofacial Surgery, UMDNJ-University Hospital/Rutgers School of Dental Medicine, Newark, New Jersey, USA \\ ${ }^{4}$ Department of Restorative Dentistry, Rutgers School of Dental Medicine, Newark, New Jersey, USA
}

Received: February 11, 2015; Accepted: March 30, 2015; Published: April 10, 2015

*Corresponding author: Mohammed E Sayed, Department of Restorative Dentistry, Rutgers School of Dental Medicine, Newark, New Jersey, USA, Tel: +1-201-406-2039; Fax: +1-973-972-0370; E-mail: drsayed203@gmail.com

\begin{abstract}
Rehabilitation of the completely edentulous patient can present a complex treatment modality in order to fulfill the needs and desires of the patient to deliver esthetic and functional prostheses. Treatment planning and sequencing for such rehabilitation often requires a multidisciplinary approach to include restorative and surgical expertise.

This clinical report will present an innovative surgical-prosthetic treatment approach utilizing an idealized complete denture setup/ duplicate to assist in appropriate implant placement, detection of any esthetic issues that need to be addressed early, jaw relation and mounting and maintenance of esthetic arrangement of teeth. Without such a well-designed, organized and integrated treatment, more severe compromises often must be made.
\end{abstract}

Keywords: Complete edentulism; Dental implants; Hybrid prosthesis; Multidisciplinary treatment; Success and survival rates

\section{Introduction}

The complete loss of teeth in one or both arches may dramatically affect the patient's mastication, esthetics, selfconfidence and social interaction [1]. Vos et al. [2], reported that edentulism affects 158 million people worldwide and is considered a chronic, non-reversible condition but proper treatment will improve the patient's quality of life. There are many ways to restore patients with complete edentulism, which include conventional complete dentures, overdentures, implant overdentures or implant supported/retained fixed prostheses. Patients treated with conventional complete dentures may be dissatisfied with this treatment outcome due to poor retention, support and stability inherent in such prostheses $[3,4]$. Fortunately, today, dental implants are considered to be an effective and successful adjunct therapy in the treatment of partial or complete edentulism. A professional team is required to manage such cases and should include a surgical specialist, a restorative dentist and a prosthetic technician. The case should be treatment planned with a collaborative team effort in order to ensure consistency and coordination in all phases of patient care, ultimately leading to proper implant placement and the delivery of final prostheses that are both functionally and esthetically successful.

Implant supported and/or retained prostheses have superior retention, stability and support, which enhance the patient's function and comfort [5]. Engfors et al. [6], reported that the cumulative survival rates of fixed implant prostheses, retained by axially placed implants were $93 \%$ in the maxilla and $99.5 \%$ in the mandible after a five year follow-up. The concept of "All on Four" has been introduced to avoid extensive ridge augmentation or grafting to develop adequate width and/or height of the ridge prior to implant placement [7]. A systematic review by Patzelt et al. [8] on the "All on Four" concept, reported a cumulative success rate of $99 \%$ after 3 years, with no difference in the success rate between axially and non-axially placed implants. This clinical report details an innovative interdisciplinary approach presenting some novel techniques for implant retained and supported complete prosthetic rehabilitation that include all phases of treatment from diagnosis through delivery of the definitive prosthesis and post-insertion follow-up.

\section{Case Report}

A 58 year old Hispanic female patient presented to postgraduate prosthodontics clinic at Rutgers School of Dental Medicine with the chief complaint that "My top bridge is not stable and I have a fetid odor from my mouth. I work as a receptionist and I'm concerned about my esthetics and social life". A review of her medical history revealed no significant findings. The dental history was taken in detail and documented in the patient's chart. A thorough prosthodontic evaluation and full set of diagnostic radiographs for comprehensive evaluation and diagnosis prior to intervention were obtained. All Maxillary teeth and the mandibular right second molar were given hopeless prognosis due to extensive carious lesions and compromised periodontal 
diagnosis (Figures 1 and 2). Smile analysis showed that the incisal edges of the maxillary anterior teeth did not follow the curvature of the lower lip (Figure 3).

Evaluation of the pre-treatment panoramic radiograph revealed adequate bone height for dental implants inferior to the maxillary sinuses bilaterally. The mandibular radiographic examination also showed horizontal bone loss of the remaining mandibular teeth and lack of vertical height in the posterior mandible bilaterally. Maxillary and mandibular irreversible hydocolloid impressions (Jeltrate Plus, Dentsply Caulk, York, $\mathrm{Pa}$ ) were made and poured in dental stone (Microstone, Whip Mix Corporation, Louisville, KY) for diagnostic casts. A record block was fabricated on the mandibular cast using visible light polymerized preformed acrylic resin sheet (Triad, Dentsply, York, Pa) and base plate wax (Modern Materials, Pearson Dental, Sylmar, CA). The vertical dimension of occlusion was evaluated using measurements of the facial proportions for esthetics [9]. The posterior speaking space was also assessed for phonetics $[10,11]$. The vertical dimension of occlusion, as presented, was found to be adequate. The maxillary cast was mounted on a semi-adjustable articulator (Hanau H2; Whip Mix, Fort Collins, CO) using a Hanau Spring-Bow record. The mandibular cast was mounted with an Alu-wax (Aluwax Dental Products CO, Allendale, Michigan) centric relation record at the proposed VDO.

The PFM crown in location of tooth \#8, which was a part of failed fixed partial denture, was chosen as a pre-extraction guide to determine the incisal edge position for setting maxillary anterior denture teeth. The mounted diagnostic casts were trimmed according to the "Rule of Thirds", to fabricate the maxillary immediate complete denture and the mandibular immediate partial denture [12]. Double cross-linked polymethylmethacrylate denture teeth were used (BlueLine, Ivoclar Vivadent, Amherst, NY). Treatment was initiated with dental and periodontal disease controlled in the preliminary phase. The patient had all remaining maxillary teeth and the mandibular right second molar extracted under local anesthesia and the sockets were grafted with a corticocancellous particulate allogenic bone graft (University of Miami Tissue Bank (UMTB); Miami, FL). The sites were dressed with collagen (Zimmer Collagen Plug; Zimmer Dental; Carlsbad, CA) and closed with 3.0 vicryl suture (Ethicon; Somerville, NJ). Maxillary and mandibular immediate prostheses were relieved and adjusted for seating over the surgical sites, and were relined using Viscogel (Dentsply International Inc., Milford, DE) (Figure 4). The immediate dentures were checked periodically during the healing period and the soft reline was changed every month. In order to further evaluate the surgical options, the patient was sent for CBCT (Cone Beam Computed Tomograph) of the maxilla and mandible with radiographic templates for both arches in place.

As discussed earlier, adequate bone quantity in the maxilla and the anterior mandible was confirmed for the placement of dental implants. Based on all presented data and the patient's desire for fixed definitive prostheses, six axial positioned implants were planned for the maxilla and the all on four technique was planned for the mandible.

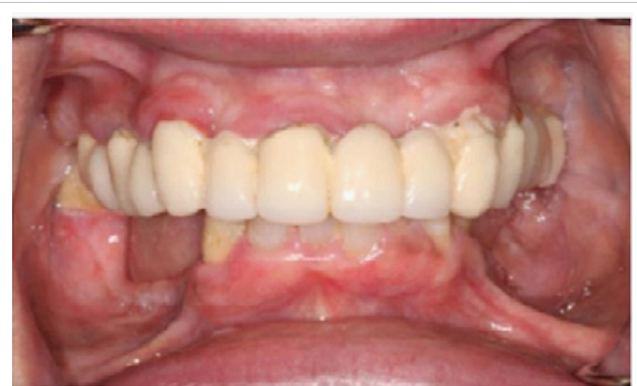

Figure 1: Pre-operative intraoral frontal view at the VDO.

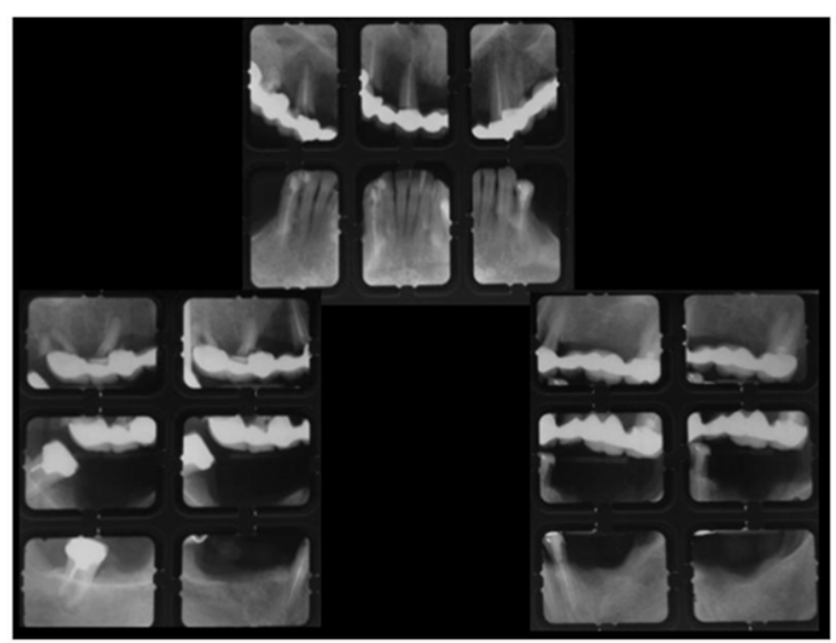

Figure 2: Pre-operative full mouth series (FMX).

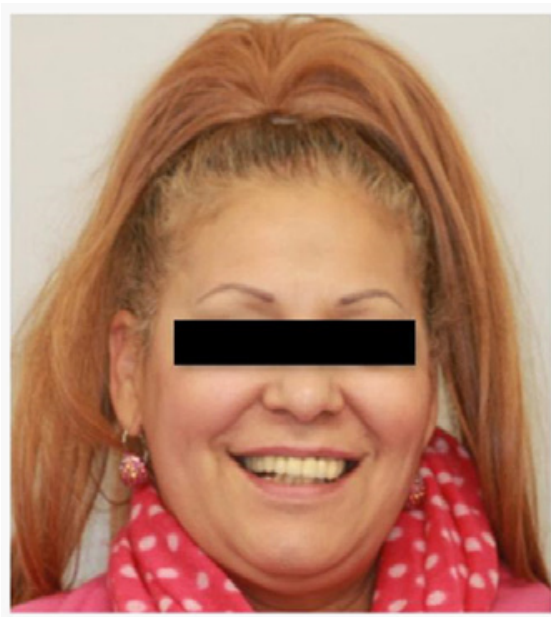

Figure 3: Pre-operative extraoral smile view.

The second surgical procedure was planned to include removal of all remaining mandibular teeth and reduction of the irregularities in the alveolar bone in order to enable the future placement of two long angled implants posteriorly and two axial positioned implants in the lateral incisor regions. The mandibular anterior teeth were extracted and a surgical template was utilized 
for the reduction of bone in the anterior mandible. The area of surgery was closed with 3.0 vicryl suture and an immediate mandibular complete denture was delivered.

Following the surgical procedure, the patient was seen at one week, two week and then again at one month postoperatively. The patient showed adequate healing and had an unremarkable postoperative course. After 3 months, the immediate denture prostheses were evaluated for esthetics and phonetics, and were found satisfactory for use as a blue print for the definitive prostheses. A treatment CBCT was taken using Dual Scan Technique in order to determine the final implant positions (Figure 5). Following confirmation of the implant position, the surgical guides were ordered with the goal of providing the patient with bone-born guided surgery for both arches. The planned maxillary implants were Nobel Active Internal-Hex 5.0 $\mathrm{x} 11.5 \mathrm{~mm}$ in the first molar regions, Nobel Active Internal-Hex $5.0 \times 10 \mathrm{~mm}$ in the second premolar regions and Nobel Active Internal-Hex $4.3 \times 13 \mathrm{~mm}$ in the canine regions. The planned mandibular implants were Nobel Active Internal-Hex 4.3 x $13 \mathrm{~mm}$ in the lateral incisor regions and Nobel Speedy Groovy ExternalHex $4.0 \times 15 \mathrm{~mm}$ in the mandibular second premolar area.

The patient presented for the planned surgical procedure which included placement of all implants using the CBCT guided surgical technique under local anesthesia. A \#15 blade was used to make a crestal incision from area of \#2 to \#15 and a full thickness mucoperiosteal flap was reflected on the buccal and palatal aspect of maxilla. The guide was placed and stabilized using four stabilizing screws. The implant sites were marked through the guide using a surgical marking pen (Devon Skin Marker; Covidien; Mansfeild, MA). The guide was then removed, and all potential implant positions were verified in relation to the crest of the ridge. The implant positions were found consistent with the preoperative surgical planning. The guide was replaced and stabilized using four stabilizing screws. Six implants were placed in the maxillary arch with the utilization of both internal and external irrigation. All implants were placed and torqued to $35 \mathrm{Ncm}$. The guide was removed and the implants were hand torqued to the crestal level. Cover screws were placed (Figure 6). The maxillary flap was repositioned and sutured with 3.0 vicryl suture.

Attention was next drawn to the mandible. A \# 15 blade was used to make a crestal incision from \# 18 to \# 31 and a full thickness mucoperiosteal flap was reflected on the buccal and lingual aspect of the mandible. The surgical guide was placed and stabilized using three stabilizing screws. The implant sites were marked through the guide using a surgical marking pen (DevonSkin Marker; Covidien; Mansfield, MA). The guide was then removed, and all potential implant positions were verified in relation with the crest of the ridge. The implant positions were found to be consistent with the preoperative surgical planning, with the exception of the angled implant at the right mandible. The marking showed the implant position to be in a position that was facial to the crest of the ridge. The decision was made to move the implant position over the crest of the ridge and to place the right mandibular angled implant without the aid of the

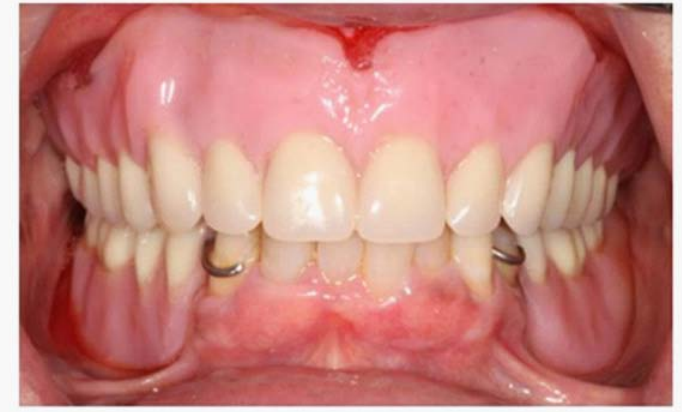

Figure 4: Intraoral view in MIP of the maxillary and mandibular immediate prostheses.

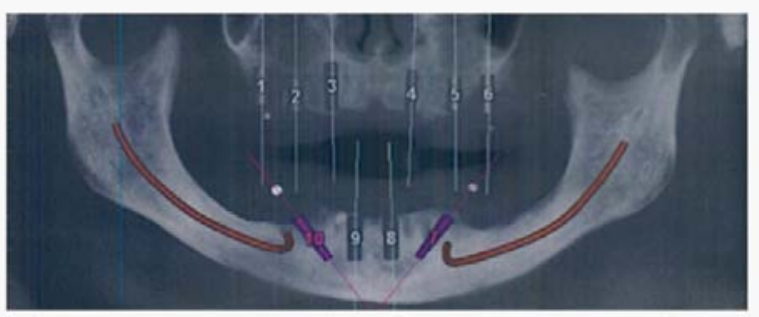

Figure 5: CBCT with virtual placement of maxillary and mandibular implants.

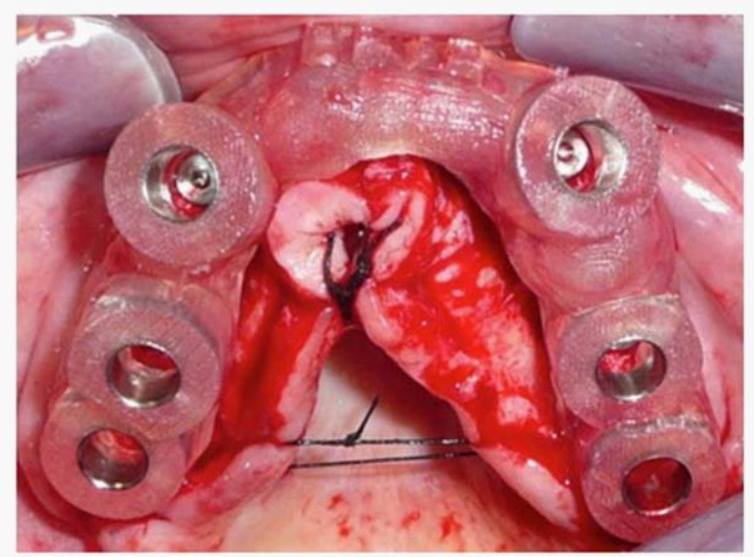

Figure 6: CBCT guided surgery for placement of 6 implants in the maxillary arch.

surgical guide. The guide was then replaced and stabilized using three stabilizing screws. Three implants were then placed in the mandibular arch with the utilization of both internal and external irrigation. These implants included the angled implant at the left mandible as well as the two axial positioned implants at the anterior mandible. The three implants were placed and torqued to $35 \mathrm{Ncm}$. The guide was then removed and the implants were hand torqued to the crestal level. Next the angled implant on 
the right mandible was placed without the aid of the surgical guide utilizing both internal and external irrigation. The implant was then torqued to $35 \mathrm{Ncm}$ to the crestal level. Cover screws were placed. The mandibular soft tissue flap was repositioned and sutured with 3.0 vicryl suture. The mandibular surgical procedure was completed with adequate hemostasis. The patient tolerated the procedure well without complication.

Immediate fixed temporization of the placed implants was not planned due to limited finance of the patient. The maxillary and mandibular immediate dentures were relined using Viscogel.

The postoperative panoramic radiograph confirmed the position of the ten implants, consistent with the pre-operative CBCT guided planning software images (Figure 7). The patient presented for follow-up visits with OMFS at one week, two weeks and one month postoperatively. The areas of surgery showed adequate healing and her postoperative course was uneventful. The maxillary and mandibular dentures were relined using Viscogel. After a period of three months, the implants were uncovered. The crestal bone level was confirmed to be at the platform level for all implants in both the maxilla and the mandible. No mobility or signs of infection were noted. Cover screws were removed and healing abutments were placed. The patient returned to the prosthodontic clinic and the complete dentures were appropriately adjusted to allow for continued usage until the determined prosthetic treatment would be accomplished.

The maxillary tooth arrangement was duplicated using Orthodontic Resin (Dentsply International Inc., Milford, DE) to be used as a custom tray and diagnostic tool. The use of the duplicate denture would also help in precise replacement of the maxillary denture teeth throughout the prosthetic treatment steps. Open tray transfer copings were attached to the maxillary implants and periapical radiographs confirmed seating of the copings. The duplicate denture was hollowed out around the copings to allow passive fit on the maxillary denture foundation area. Ultralow viscosity polyvinylsiloxane impression material (Aquasil, Dentsply, Detrey GmbH, Konstanz, Germany) was injected around the copings to capture the soft tissue details. The duplicate denture was then filled with high viscosity polyvinylsiloxane impression material and seated in patient's mouth and removed after 7 minutes. It was noted that the implant in position \#11 was projecting labially through the duplicate denture, which presented a problem for the design of the maxillary definitive prosthesis (Figure 8).

Open tray transfer copings were attached to the axially placed mandibular anterior implants. Multiunit abutments were attached to mandibular distally angulated implants to attain a path of insertion consistent with the mandibular anterior implants. Transfer copings were attached to the multiunit abutments and periapical radiographs were taken to verify seating of the copings. The final mandibular impression was made using the same procedure as the maxillary arch.

The maxillary and mandibular impressions were supported with laboratory putty (Lab Putty, Coltène/Whaledent Inc.,

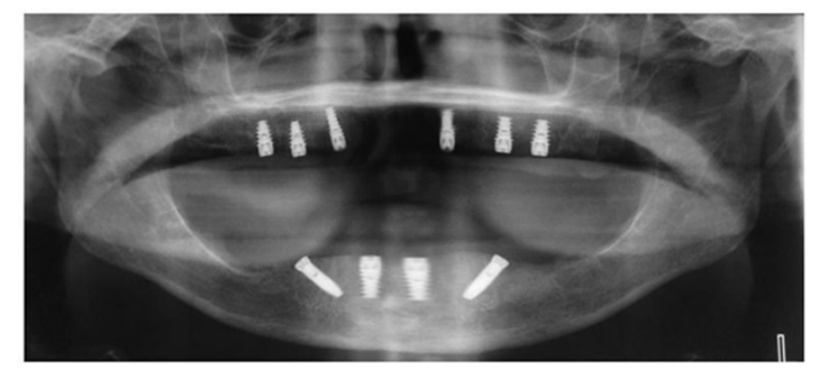

Figure 7: Panoramic radiograph after implant placement.

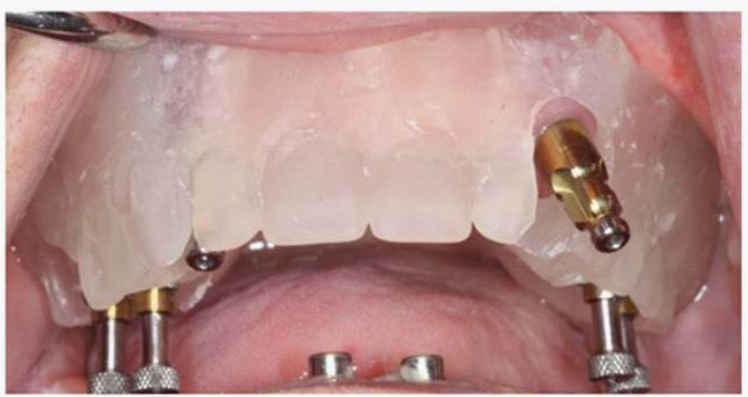

Figure 8: Intraoral view of the maxillary duplicate denture. Implant \#11 presented significant facial projection.

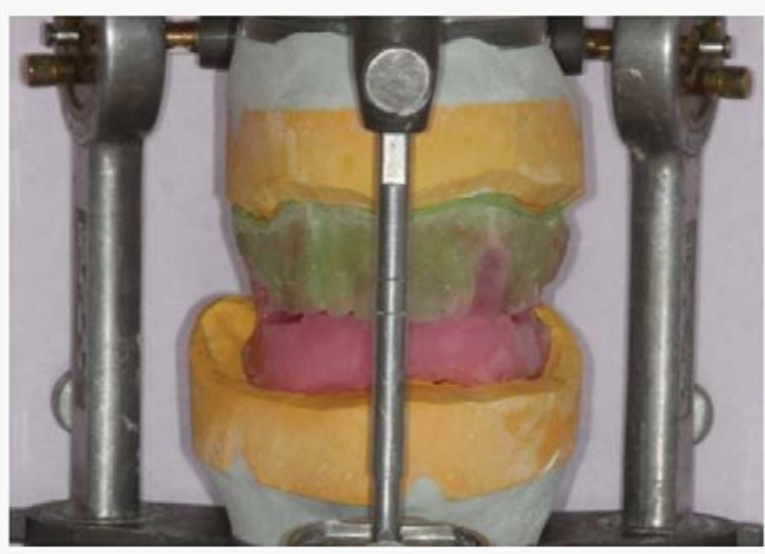

Figure 9: Maxillary denture duplicate to mount the maxillary master cast.

Cuyahoga Falls, $\mathrm{OH}$ ) to prevent excessive pressure on the transfer copings, which might distort the final impression. A silicon material (Softissue Moulage, Kerr Corporation, Orange, CA) was injected around the implant transfer copings/replicas. Both impressions were beaded, boxed and poured in low expansion and high strength dental stone (Silky-Rock, Whip Mix Corporation). The maxillary duplicate denture that was used for the final impression was carefully retrieved from the stone cast and was used as a record base to mount the maxillary master cast. A mandibular screw-retained record base was fabricated. 


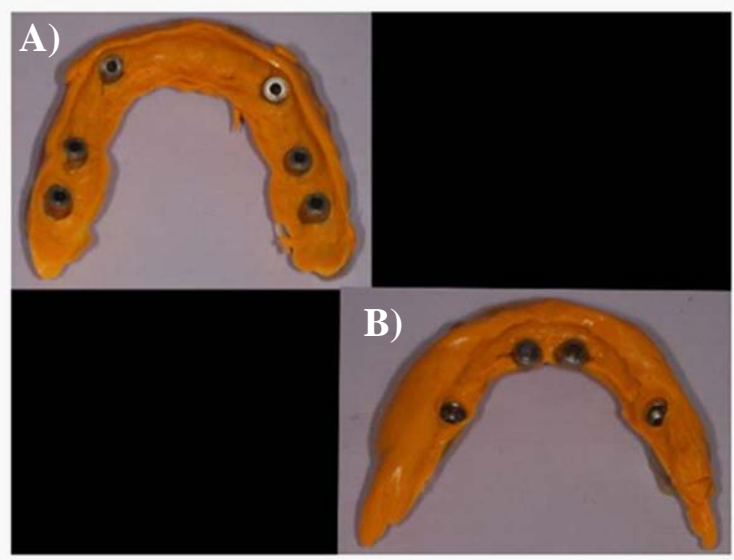

Figure 10: Tissue side of the ployvinylsiloxane-waxed (A) maxillary and (B) mandibular hybrid prostheses.

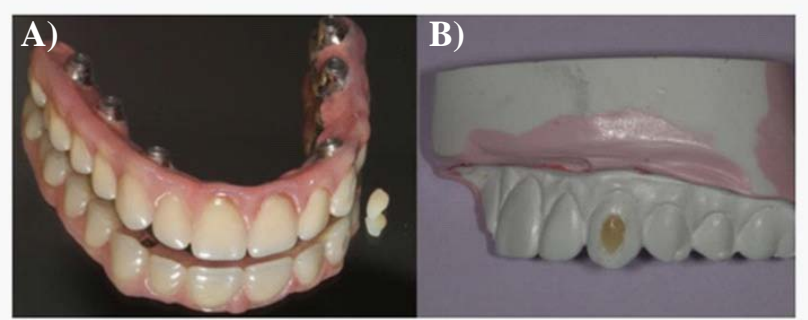

Figure 11: (A) Finished maxillary hybrid prosthesis with laboratory processed composite plug. (B) Epoxy resin model with replacement composite plug.

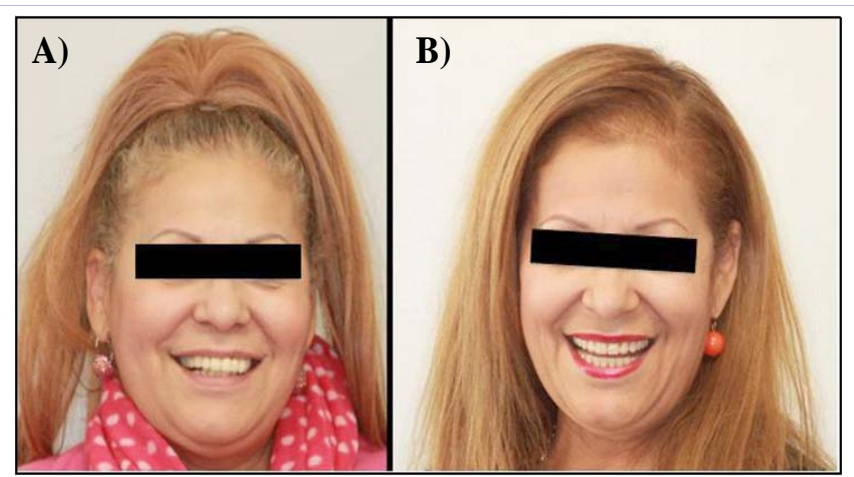

Figure 12: Extraoral smile views (A) before and (B) after completion of treatment.

Face-bow transfer and centric relation records were made at the VDO and the maxillary and mandibular master casts were mounted on Hanau semi-adjustable articulator (Figure 9). A labial putty index was made of the mounted duplicate maxillary denture for precise replacement of the maxillary tooth position and arrangement. A new maxillary denture base was fabricated. The labial putty index was used to arrange the maxillary denture teeth in the exact positions and arrangement of the teeth on the duplicate maxillary denture. The same denture tooth mold and shade were used for the definitive prostheses. Screw retained maxillary and mandibular trial denture bases were checked intra-orally to record centric relation and confirm the vertical dimension of occlusion, esthetics and phonetics. A new labial putty index was made of the mounted trial dentures to guide in construction of the metal frameworks. The metal frameworks were waxed, cast, finished and tried in the patient's mouth for passivity of fit using the one-screw test technique [13]. Periapical radiographs verified seating of the frameworks. The denture teeth were re-arranged on metal framework using the labial putty index. The denture tooth in position \#11 was drilled labially to accommodate the abutment screw access. Maxillary and mandibular waxed hybrid prostheses were tried in to verify the VDO, centric relation, esthetics, phonetics and occlusion. At the try-in stage of both arches, a space was noted between the metal framework and alveolar ridges. An Ultra-low viscosity polyvinylsiloxane impression material was injected below the metal framework to record the soft tissue covering the maxillary and mandibular alveolar ridges (Figure 10). The master casts were trimmed to allow for complete seating of the polyvinylsiloxanewaxed prostheses. A Soft tissue Moulage was injected to fill the gap between the impression and the cast to form the contour of the alveolar ridges. The spaces below the metal frameworks were filled with wax and the hybrid prostheses were waxed-up, festooned and processed in heat-cured polymethyl-methacrylate (Lucitone 199, Dentsply). After processing, a laboratory remount was performed to compensate for processing errors. The prostheses were finished and polished.

An angle correction abutment for implant \#11 was avoided due to vertical space deficiency.

An impression of the polished maxillary hybrid prosthesis was made in laboratory putty and poured in epoxy-resin (Resin-Rock, Whip Mix Corporation). A Composite plug (Gradia, Light-Cured Micro-Ceramic Composite, GC America, Inc, Alsip, IL) was used to seal the labial screw access hole for implant \#11 to maintain an optimum esthetic outcome. The plug was fabricated on the epoxy resin model. Replacement plugs were made, archived and made accessible for replacement in case maxillary hybrid prosthesis had to be removed for maintenance or repair (Figure 11). The abutment screws were torqued to $35 \mathrm{Ncm}$. The screw holes were filled with Ultra-low viscosity polyvinylsiloxane impression material (Aquasil, Dentsply, Detrey GmbH, Konstanz, Germany) and sealed with light cured resin material (Fermit, Ivoclar North America, Ontario, Canada). The occlusion was checked for possible interferences after using light cure resin to seal the screw holes (Figure 12). Oral hygiene instructions were given with manual demonstration. Recall visits were scheduled after 1 week, 1 month and every 3-4 months for the first year then every 6 months thereafter.

\section{Conclusion}

Multidisciplinary treatment planning is very critical for management of complete oral rehabilitation cases. The use of advanced imaging technology is an adjunct for successful dental implant placement. However, these technologies can present with some built in errors, which lead to compromised 
implant placement. These compromises should be identified and addressed early in prosthetic treatment steps and solutions to be explored.

\section{Conflict of Interest}

The authors declare that there is no conflict of interest of any kind for publishing this paper.

\section{References}

1. Rosenbaum N. Full-arch implant-retained prosthetics in general dental practice. Dent Update. 2012; 39 (2):108-10.

2. Vos T, Flaxman AD, Naghavi M, Lozano R, Michaud C, Ezzati M, et al. Years Lived with Disability (YLDs) for 1160 sequelae of 289 diseases and injuries 1990-2010: a systematic analysis for the Global Burden of Disease Study 2010. Lancet. 2012; 380(9859): 2163-96.

3. Carlsson GE, Otterland A, Wennström A, Odont D. Patient factors in appreciation of complete dentures. J Prosthet Dent. 1967; 17(4): 322328

4. Awad MA, Feine JS. Measuring patient satisfaction with mandibular prostheses. Community Dent Oral Epidemiol. 1998; 26(6): 400-405.

5. Awad MA, Lund JP, Dufresne E, Feine JS. Comparing the efficacy of mandibular implant-retained overdentures and conventional dentures among middle- aged edentulous patients: satisfaction and functional assessment. Int J Prosthodont. 2003; 16(2): 117-122.

6. Engfors I, Ortorp A, Jemt T. Fixed implant supported prostheses in elderly patients: a 5-year retrospective study of 133 edentulous patients older than 79 years. Clin Implant Dent Rel Res. 2004; 6(4): 190-198.

7. Aparicio C, Perales $\mathrm{P}$, Rangert B. Tilted implants as an alternative to maxillary sinus grafting: a clinical, radiologic, and periotest study. Clin Implant Dent Relat Res. 2001; 3(1): 39-49.

8. Patzelt SB, Bahat O, Reynolds MA, Strub JR. The All-on-Four Treatment Concept: A Systematic Review. Clin Implant Dent Relat Res. 2013; 16(6): 836-55

9. Bowman AJ, Chick AO. A Note on Facial Proportions. Br Dent J. 1962; 112: 288-289.

10. Silverman MM. The speaking method in measuring vertical dimension. J Prosthet Dent. 2001; 85(5):427-31.

11. Pound E. Let /S/ be your guide. J Prosthet Dent. 1977; 38(5):482-9.

12. Jerbi FC. Trimming the cast in the construction of immediate dentures. J Prosthet Dent. 1966; 16(6):1047-53.

13. Kan JY, Rungcharassaeng K, Bohsali K, Goodacre CJ, Lang BR. Clinical methods for evaluating implant framework fit. J Prosthet Dent. 1999 . 81(1): 7-13. 